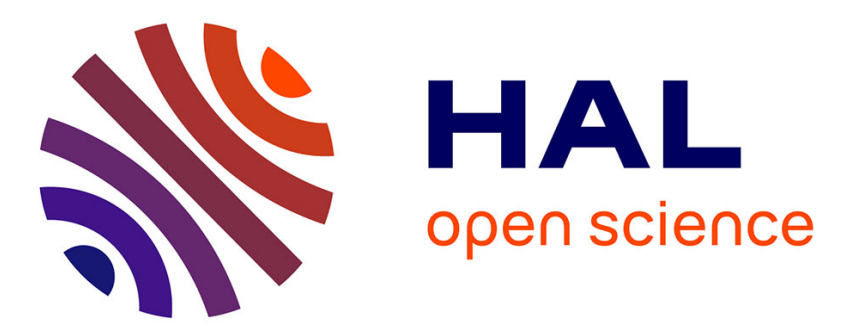

\title{
Multiple oscillatory modes of the Argentine basin. Part II: The spectral origin of basin modes
}

Wilbert Weijer, Frédéric Vivier, Sarah T. Gille, Henk A. Dijkstra

\section{To cite this version:}

Wilbert Weijer, Frédéric Vivier, Sarah T. Gille, Henk A. Dijkstra. Multiple oscillatory modes of the Argentine basin. Part II: The spectral origin of basin modes. Journal of Physical Oceanography, 2007, 37, pp.2869-2881. 10.1175/2007JPO3688.1 . hal-00770721

\section{HAL Id: hal-00770721 \\ https://hal.science/hal-00770721}

Submitted on 10 Jun 2021

HAL is a multi-disciplinary open access archive for the deposit and dissemination of scientific research documents, whether they are published or not. The documents may come from teaching and research institutions in France or abroad, or from public or private research centers.
L'archive ouverte pluridisciplinaire HAL, est destinée au dépôt et à la diffusion de documents scientifiques de niveau recherche, publiés ou non, émanant des établissements d'enseignement et de recherche français ou étrangers, des laboratoires publics ou privés. 


\title{
Multiple Oscillatory Modes of the Argentine Basin. Part II: The Spectral Origin of Basin Modes
}

\author{
WILBERT WEIJER \\ Scripps Institution of Oceanography, La Jolla, California, and Los Alamos National Laboratory, Los Alamos, New Mexico \\ FRÉDÉRIC VIVIER \\ Laboratoire d'Océanographie et du Climat: Expérimentation et Approches Numériques, Paris, France
}

SARAH T. GiLle

Scripps Institution of Oceanography, La Jolla, California

Henk A. DijKstra

Institute for Marine and Atmospheric Research Utrecht, Utrecht University, Utrecht, Netherlands

(Manuscript received 31 August 2006, in final form 11 December 2006)

\begin{abstract}
In this paper the spectrum of barotropic basin modes of the Argentine Basin is shown to be connected to the classical Rossby basin modes of a flat-bottom (constant depth), rectangular basin. First, the spectrum of basin modes is calculated for the Argentine Basin, by performing a normal-mode analysis of the barotropic shallow-water equations. Then a homotopy transformation is performed that gradually morphs the full-bathymetry geometry through a flat-bottom configuration into a rectangular basin. Following the eigenmodes through this transition establishes a connection between most of the basin modes and the classical Rossby basin modes of a rectangular geometry. In particular, the 20-day mode of the Argentine Basin is identified with the lowest-order mode of classical theory. Sensitivity studies show that the decay rate of each mode is controlled by bottom friction, but that it is insensitive to lateral friction; lateral friction strongly impacts the oscillation frequency. In addition, the modes are found to be only slightly sensitive to the presence of a background flow.
\end{abstract}

\section{Introduction}

In the first part of this study (Weijer et al. 2007, hereinafter Part I), statistical analyses of altimeter data suggested that more than one mode of variability may be present in the Argentine Basin. This conclusion may reconcile several studies reporting on modes of variability with conflicting periods (Weatherly 1993; Fu et al. 2001; Hughes et al. 2007). It was also found that some of the modes identified in altimeter data were consistent in their oscillation period and spatial pattern

Corresponding author address: Dr. Wilbert Weijer, CCS-2, MS B296, Los Alamos National Laboratory, Los Alamos, NM 87545. E-mail: wilbert@lanl.gov with barotropic basin modes of the Argentine Basin, determined for a shallow-water context. In fact, a normal-mode analysis revealed an entire spectrum of such basin modes.

The purpose of this paper is to understand the physics of propagation of the Argentine Basin modes. Instead of analyzing their dynamical balances in detail, we will search for connections between these modes and modes whose dynamics are well known: classical Rossby basin modes in a rectangular, flat-bottom basin. Theoretical considerations indicate that closed basins with idealized geometry exhibit a spectrum of planetary wave modes, also known as Rossby basin modes (Longuet-Higgins 1964). These basin modes are superpositions of Rossby waves that satisfy the boundary

DOI: $10.1175 / 2007 J P O 3688.1$

(C) 2007 American Meteorological Society 
conditions of a basin. If sea surface variations can be ignored in the vorticity balance (small Froude number; justified for deep or small basins), the streamfunction $\Psi_{(n, m)}$ of a normal mode of a rectangular basin with dimensions $L_{1} \times L_{2}$ consists of a carrier wave and an envelope of sine functions:

$$
\psi_{(n, m)}=\cos \left[\frac{\beta_{0} x}{2 \sigma_{(n, m)}}+\sigma_{(n, m)} t\right] \sin \frac{n \pi x}{L_{1}} \sin \frac{m \pi y}{L_{2}},
$$

where $x$ and $y$ are the zonal and meridional coordinates, $t$ is time, $\beta_{0}$ is the gradient of planetary vorticity, and $\sigma_{(n, m)}$ is the frequency of the mode with mode number $(n, m)$. Its period is given by

$$
T_{(n, m)}=\frac{4 \pi}{\beta_{0}}\left[\left(\frac{n \pi}{L_{1}}\right)^{2}+\left(\frac{m \pi}{L_{2}}\right)^{2}\right]^{1 / 2} .
$$

There is a fundamental equivalence between gradients in planetary vorticity and bathymetry in the vorticity balance of barotropic fluids. This equivalence can be made explicit by expressing the gradient of potential vorticity $f / H$ in terms of an "effective $\beta$ " field, $\beta^{*}=$ $\beta_{0}-f \nabla \ln H$, where $H$ is the variable water depth (Ripa 1978). In fact, when one considers the basin modes (1) as eigenvectors of the barotropic vorticity equation (formulated in terms of streamfunction $\psi$; Pedlosky 1987),

$$
\frac{\partial}{\partial t}\left(\nabla^{2} \psi\right)+\beta_{0} \frac{\partial \psi}{\partial x}=0
$$

it is clear that for reasonable choices of bathymetry the "dynamical core" of this equation does not fundamentally change when $\beta_{0}$ is replaced by $\beta^{*}$. This suggests that the spectrum of basin modes of the flat-bottom configuration should carry over to nonflat geometries, and that the physics of propagation is invariant under such a transformation (unless strong interaction between modes occur). So tracing a mode in a realistic basin to its mode of origin from classical theory (determining its "spectral origin") will provide a clue about its mechanism of propagation.
TABLE 1. Model parameters and their standard values.

\begin{tabular}{llc}
\hline \hline Symbol & \multicolumn{1}{c}{ Scales } & \multicolumn{1}{c}{ Value } \\
\hline$H_{0}$ & Equilibrium depth & $6288.6 \mathrm{~m}$ \\
$r_{0}$ & Earth's radius & $6.37 \times 10^{6} \mathrm{~m}$ \\
$\Omega$ & Earth's rotation rate & $7.272 \times 10^{-5} \mathrm{~s}^{-1}$ \\
$g$ & Gravitational constant & $9.8 \mathrm{~m} \mathrm{~s}^{-2}$ \\
$\rho$ & Density & $1020 \mathrm{~kg} \mathrm{~m}^{-3}$ \\
$A_{h}$ & Horizontal viscosity & $3.0 \times 10^{3} \mathrm{~m}^{2} \mathrm{~s}^{-1}$ \\
$r$ & Bottom friction & $3.64 \times 10^{-6} \mathrm{~s}^{-1}$ \\
\hline
\end{tabular}

In this paper we will attempt to establish connections between the basin modes of the Argentine Basin and Rossby basin modes of classical theory. To that end, we will perform a "homotopy" transformation that smoothly transforms the Argentine Basin into a flatbottom rectangular domain. The eigenmodes will be traced during this transformation. When the limit of the rectangular domain is reached, the resulting modes will be compared to the patterns and oscillation periods of the classical modes [i.e., (1) and (2)].

A similar procedure will be applied to estimate the sensitivity of these basin modes with respect to friction, domain size, and background circulation. Tracing eigenmodes through parameter space has led to illuminating results regarding tropical ocean-atmosphere variability (Jin and Neelin 1993), low-frequency variability of the wind-driven circulation (Simonnet and Dijkstra 2002), and variability of the overturning circulation (Dijkstra 2006).

\section{Barotropic Rossby basin modes of the Argentine Basin}

\section{a. The shallow-water model}

We study the normal modes of the Argentine Basin using a barotropic shallow-water (SW) model. It is based on the code used by Schmeits and Dijkstra (2000), but includes full bathymetry and bottom friction. The set of equations is given by

$$
\begin{aligned}
& \frac{\partial u}{\partial t}+\frac{u}{r_{0} \cos \phi} \frac{\partial u}{\partial \lambda}+\frac{v}{r_{0}} \frac{\partial u}{\partial \phi}-\frac{u v}{r_{0}} \tan \phi-2 \Omega v \sin \phi=-\frac{g}{r_{0} \cos \phi} \frac{\partial \eta}{\partial \lambda}+A_{h}\left(\nabla^{2} u-\frac{u}{r_{0}^{2} \cos ^{2} \phi}-\frac{2 \sin \phi}{r_{0}^{2} \cos ^{2} \phi} \frac{\partial v}{\partial \lambda}\right)+\frac{\tau^{\lambda}}{\rho_{0} h}-r u, \\
& \frac{\partial v}{\partial t}+\frac{u}{r_{0} \cos \phi} \frac{\partial v}{\partial \lambda}+\frac{v}{r_{0}} \frac{\partial v}{\partial \phi}+\frac{u^{2}}{r_{0}} \tan \phi+2 \Omega u \sin \phi=-\frac{g}{r_{0}} \frac{\partial \eta}{\partial \phi}+A_{h}\left(\nabla^{2} v-\frac{v}{r_{0}^{2} \cos ^{2} \phi}+\frac{2 \sin \phi}{r_{0}^{2} \cos ^{2} \phi} \frac{\partial u}{\partial \lambda}\right)+\frac{\tau^{\phi}}{\rho_{0} h}-r v, \quad(5)
\end{aligned}
$$

and $\frac{\partial \eta}{\partial t}+\frac{1}{r_{0} \cos \phi}\left[\frac{\partial(h u)}{\partial \lambda}+\frac{\partial(h v \cos \phi)}{\partial \phi}\right]=0$.
Here $(u, v)$ are the depth-averaged zonal and meridional velocity components; $h=H_{0}+\eta-h_{b}$ is total 


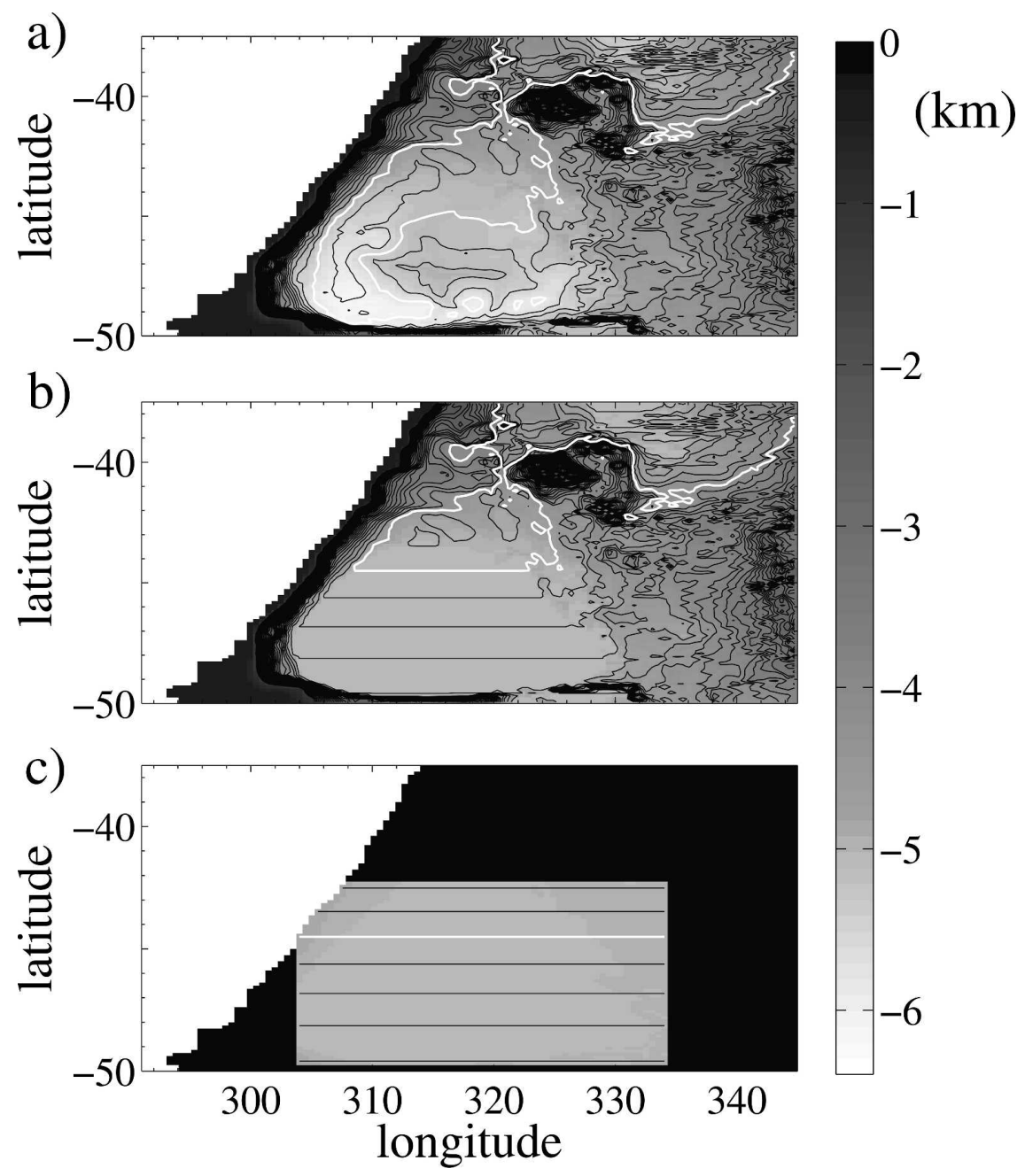

FIG. 1. End-member bathymetries used in the normal-mode analysis: (a) full bathymetry $H_{\text {full }}$, (b) flat-bottom Argentine Basin $H_{\text {flat }}$, and (c) rectangular basin $H_{\text {rect }}$. Gray shading denotes water depth $(\mathrm{km})$; contours show $\log (f / h)$, plotted for the interval $[-8.0,-7.0]$ with step 0.02 . White contour denotes -7.74 value.

water depth, with $H_{0}$ denoting maximum equilibrium depth, $\eta$ denoting sea surface elevation, and $h_{b}$ denoting bathymetry; $\lambda$ and $\phi$ are east longitude and latitude, respectively, expressed in radians; and $\tau^{\lambda}$ and $\tau^{\phi}$ denote zonal and meridional wind stress. In this paper, only unforced solutions are considered $\left(\tau^{\lambda}=\tau^{\phi}=0\right)$, but realistic wind stress is used in Part I for transient integrations. Model parameters and their values are tabulated in Table 1.

The model domain covers the region between $50^{\circ}$ and $25^{\circ} \mathrm{S}, 291^{\circ}$ and $345^{\circ} \mathrm{E},-$ and includes the Argentine Basin. The numerical grid consists of $106 \times 100$ grid points, which is equivalent to a spatial resolution of $0.5^{\circ} \times 0.25^{\circ}$. Bathymetry is based on the 2-minute gridded elevations/bathymetry for the world (ETOPO2) dataset, box-averaged onto our model grid (Fig. 1a).
Depths smaller than $300 \mathrm{~m}$ (the continental shelf) are set to zero.

The discretized SW equations can be expressed as

$$
\mathbf{M} \frac{\partial \mathbf{x}}{\partial t}+\mathbf{L x}+\mathbf{N}(\mathbf{x})=0
$$

where $\mathbf{x}$ denotes the state vector, $\mathbf{M}$ denotes the mass matrix, $\mathbf{L}$ denotes the linear operators, and $\mathbf{N}$ denotes the nonlinear operators. Let $\overline{\mathbf{x}}$ be a steady solution of this system, and let $\tilde{\mathbf{x}}$ be a small perturbation such that $\mathbf{x}=\overline{\mathbf{x}}+\tilde{\mathbf{x}}$. Neglecting terms quadratic in the perturbations, we get

$$
\mathbf{M} \frac{\partial \tilde{\mathbf{x}}}{\partial t}+\mathbf{L} \tilde{\mathbf{x}}+\mathbf{N}_{x}(\overline{\mathbf{x}}) \tilde{\mathbf{x}}=0
$$


a) Mode 1
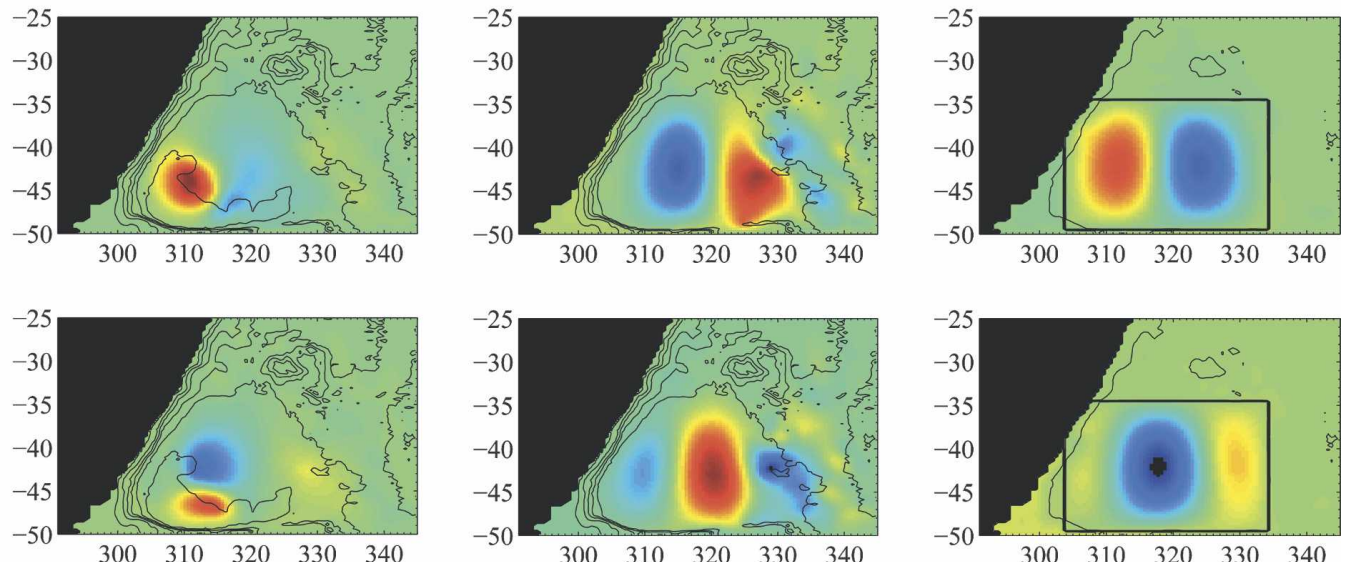

b) Mode 2
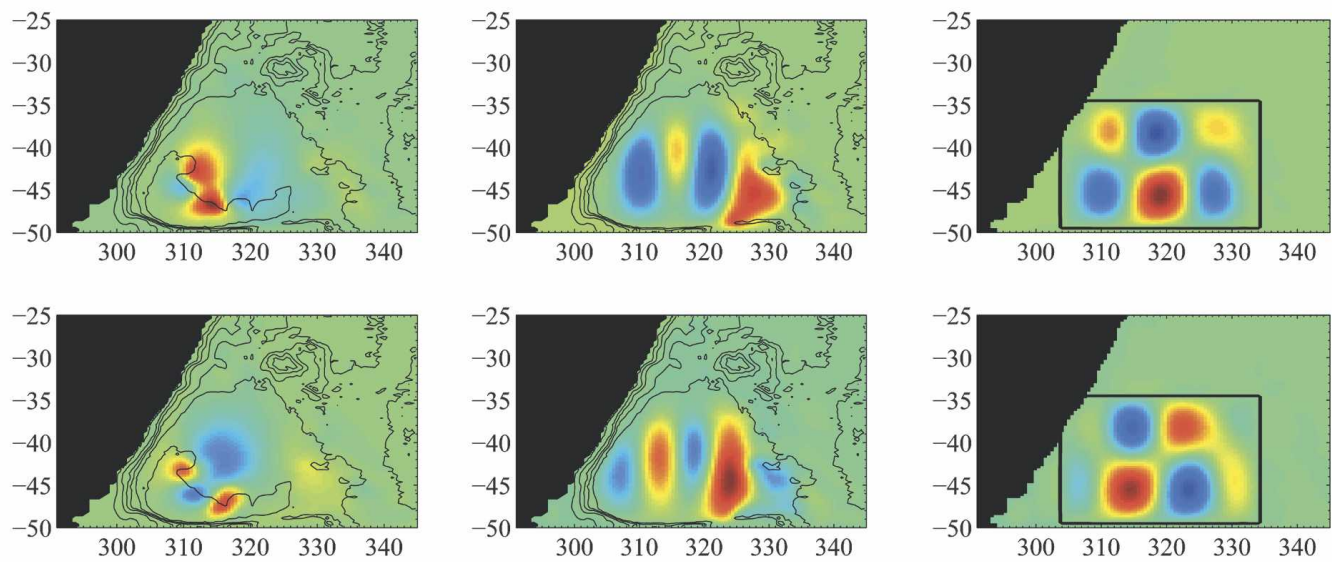

c) Mode 3
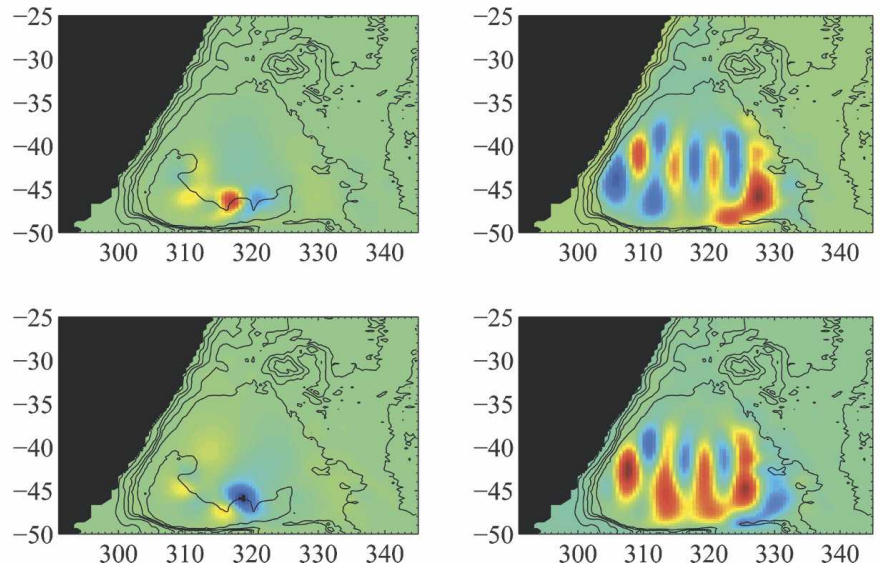

FIG. 2. Spatial patterns of the Rossby basin modes for (left) full-bathymetry $[(\alpha, \beta)=(0,0)]$, (center) flat-bottom $[(\alpha, \beta)=(1,0)]$, and (right) rectangular basin $[(\alpha, \beta)=(1,1)]$. Upper plot in each panel shows the real part of the mode $\left(\hat{\mathbf{x}}_{r}\right)$; lower plot shows the (minus) imaginary part $\left(-\hat{\mathbf{x}}_{i}\right)$. Time sequence goes as follows: $\hat{\mathbf{x}}_{r} \rightarrow-\hat{\mathbf{x}}_{i} \rightarrow-\hat{\mathbf{x}}_{r} \rightarrow$ $\hat{\mathbf{x}}_{i}$. (a) Mode 1, (b) mode 2, (c) mode 3, (d) mode 4, (e) mode 5, and (f) mode 6. Note that for mode 3 no equivalent rectangular Rossby basin mode could be found. Units are arbitrary; color scale ranges from blue $(-\max |\eta|)$ to red $(\max |\eta|)$, and centers around green (0). 
d) Mode 4
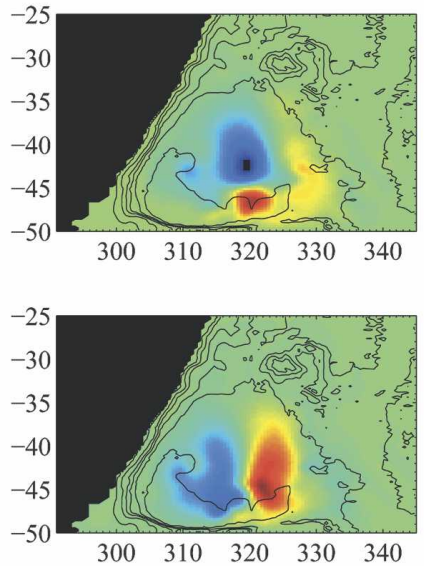

e) Mode 5
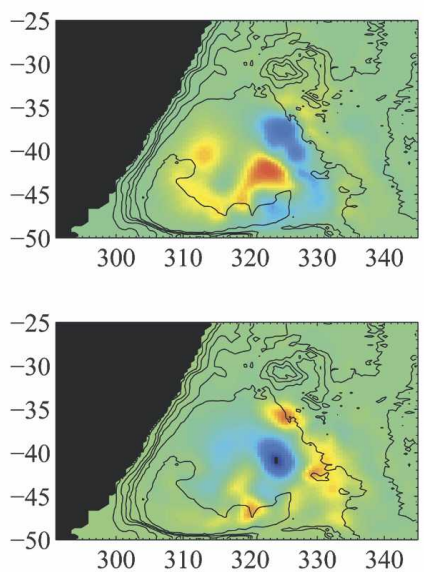

f) Mode 6
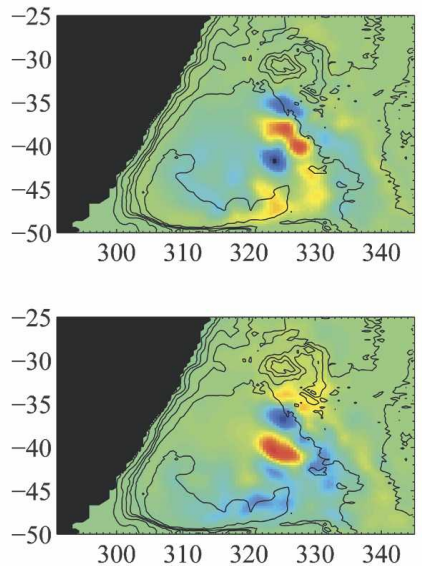
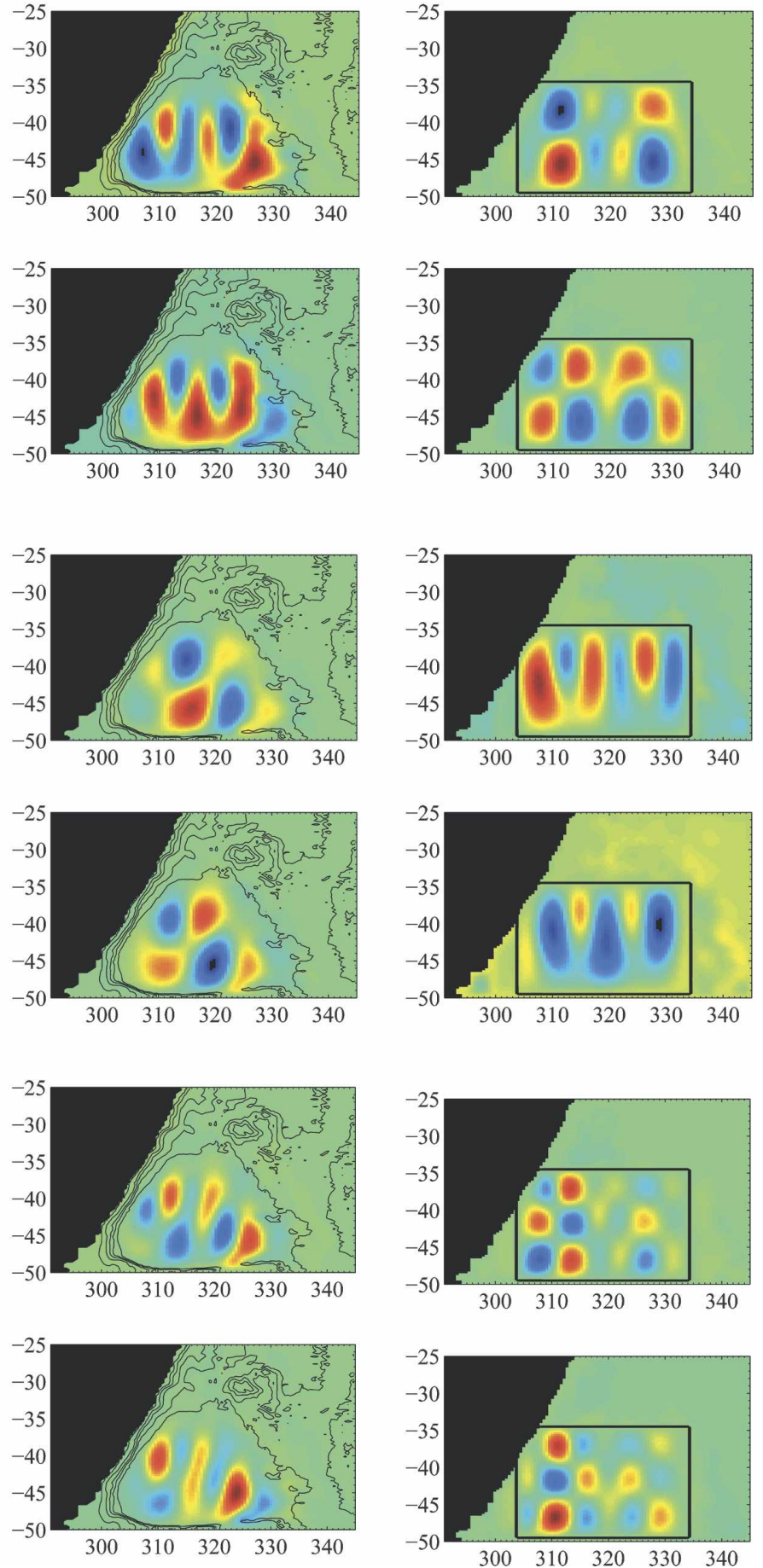

FIG. 2. (Continued)

where $\mathbf{L}+\mathbf{N}_{x} \equiv \mathbf{A}$ is the Jacobian matrix. Inserting the generic form $\tilde{\mathbf{x}}=\hat{\mathbf{x}} e^{\sigma t}$ leads to a generalized eigenvalue problem of the form

$$
\mathbf{A} \hat{\mathbf{x}}=\sigma \mathbf{B} \hat{\mathbf{x}}
$$

where $\mathbf{B}=-\mathbf{M}$. Here, $\sigma=\sigma_{r}+i \sigma_{i}$ is the complex growth factor: the decay time scale of $\tilde{\mathbf{x}}$ (and hence the linear stability of the state $\overline{\mathbf{x}}$ ) is determined by its real part according to $1 / \sigma_{r}$, while the oscillation period is given by $2 \pi / \sigma_{i}$. The eigenvectors $\hat{\mathbf{x}}$ for each $\sigma$ are called 
the eigenmodes associated with $\overline{\mathbf{x}}$. If $\sigma_{i} \neq 0$ the mode is oscillatory and these eigenmodes are complex: $\hat{\mathbf{x}}=\hat{\mathbf{x}}_{r}+$ $i \hat{\mathbf{x}}_{i}$. The oscillation proceeds as $\hat{\mathbf{x}}_{r} \rightarrow-\hat{\mathbf{x}}_{i} \rightarrow-\hat{\mathbf{x}}_{r} \rightarrow \hat{\mathbf{x}}_{i} \rightarrow \ldots$

For most of this study, a motionless background solution $\overline{\mathbf{x}}$ is considered. This assumption renders $\mathbf{N}_{x}(\overline{\mathbf{x}})=$ 0 and basically eliminates all nonlinear advective terms, as well as nonlinear inertial terms that arise from the spherical geometry.

A powerful method to solve the generalized eigenvalue problem (9) is the Jacobi-Davidson "QZ" method (JDQZ; Sleijpen and Van der Vorst 1996). This method calculates eigenvalues that are closest to a prespecified target value. Once an eigenvalue has been found, and the corresponding eigenvector has been determined, one can easily trace the evolution of this eigenmode versus model parameters.

In this study we want to find out whether the fullbathymetry modes are related to the classical Rossby basin modes of a corresponding rectangular basin. Establishing a connection between the spectrum of the full-bathymetry case and the countable set of modes from classical theory would assign a "spectral origin" to each of the modes, and would provide useful information about the number of modes that can be expected. To establish this link, we will transform the basin geometry from the full-bathymetry case $\left(H_{\text {full }}\right.$; Fig. 1a) via a flat-bottom (constant depth) Argentine Basin $\left(H_{\text {flat }}\right.$; Fig. 1b) to a rectangular configuration $\left(H_{\text {rect }} ;\right.$ Fig. 1 c). The gradual transition will be governed by so-called homotopy parameters $\alpha$ and $\beta$, through

$$
H=(1-\beta)\left[(1-\alpha) H_{\text {full }}+\alpha H_{\text {flat }}\right]+\beta H_{\text {rect }} .
$$

We calculate a prespecified number of modes (usually 8) at fixed values of $\alpha$ and $\beta$ between 0 and 1. By following the growth factor and angular frequency, the evolution of individual modes can be traced. The complex eigenvalues $\sigma=\sigma_{r}+i \sigma_{i}$ usually vary smoothly with $\alpha$ and $\beta$, and the pairs $\left(\sigma_{r}, \sigma_{i}\right)$ form distinct clusters for each mode that facilitate identification. When in doubt, the spatial patterns clearly identify the modes, making this process unambiguous.

\section{b. Rossby basin modes of the Argentine Basin}

For the full-bathymetry Argentine Basin, seven eigenmodes are found with periods below 50 days. We will focus on the six modes that can be identified as Rossby basin modes. As shown by Fig. 2 (left panels), these modes differ considerably in their spatial patterns. Modes 1 to 4 all display rotating multipole structures. Modes 1, 3, and 4 are characterized by a bipolar pattern, which is either large-scale (modes 1 and 4) or small-scale (mode 3$)$. Mode 2 features a quadrupole structure. Modes 5 and 6 are most prominent in the eastern part of the Argentine Basin. They feature anomalies that appear to propagate southward.

Figure 3 shows the temporal evolution of the first four modes in the full-bathymetry case. It is clear that the propagation of these modes is strongly controlled by the contours of $f / H$, which appear to form waveguides. Mode 1 is strongly governed by the semicircular shape of the $f / H$ contours on the western flank of the Zapiola Rise. Mode 2 appears to be a higher-order mode captured by this same dynamical feature. In contrast, mode 4 is controlled by the semicircular $f / H$ contours on the eastern flank of the Zapiola Rise, while mode 3 seems to propagate along a small circular contour southeast of the Zapiola Rise. Interestingly, neither of these modes go totally around the Zapiola Rise. In particular, both modes 1 and 4 seem to have a rapid "short-circuiting" phase that transports anomalies more or less over the Zapiola Rise.

For the current choice of parameter values, the decay time scale $T_{\text {decay }}$ of these basin modes is about 3 days (Table 2). The oscillation periods $T_{\text {full }}$ range from about 20 days for mode 1 to 45 days for mode 6 .

\section{c. The spectral origin of the basin modes}

The transformation of the Argentine Basin to a rectangular configuration takes place in two steps. First, the bathymetry of the Argentine Basin is flattened out (through the parameter $\alpha$ ) until the flat-bottom geometry $H_{\text {flat }}$ is reached. Then, the flat-bottom Argentine Basin is transformed (through $\beta$ ) into a rectangular geometry, $H_{\text {rect }}$ (Fig. 4b). The end point, $\beta=1$, could not be reached due to the depths surrounding the basin becoming zero, but $\beta=0.97$ is close enough to enable a consistent description.

As shown by Fig. 4, each of the modes 1 through 6 can be followed to the flat-bottom domain, and, except for mode 3 , to the rectangular geometry. As is evident from Fig. 2, the transformation of the basin modes is striking. Most modes evolve from relatively small-scale, often rotating structures into basin-filling, westward propagating signals. Mode 3, for example, evolves from a small-scale structure around the Zapiola Rise with a period below 30 days to a basin-filling oscillation with a period exceeding 60 days for $H_{\text {flat }}$ (Table 2).

The spatial structures of the modes for $\beta \approx 1$ (Fig. 2; right columns) can be compared to the patterns of classical Rossby basin modes of rectangular basins, Eq. (1). Table 2 shows the likely mode numbers $(n, m)$ based on this comparison, the periods $T_{(n, m)}$ predicted from Eq. (2), and the actual period of the modes calculated for $\beta \approx 1\left(T_{\text {rect }}\right)$. As a matter of fact, the calculations for $\beta \approx 1$ reveal the entire spectrum of lowest-order classical basin modes (Table 3 ). 

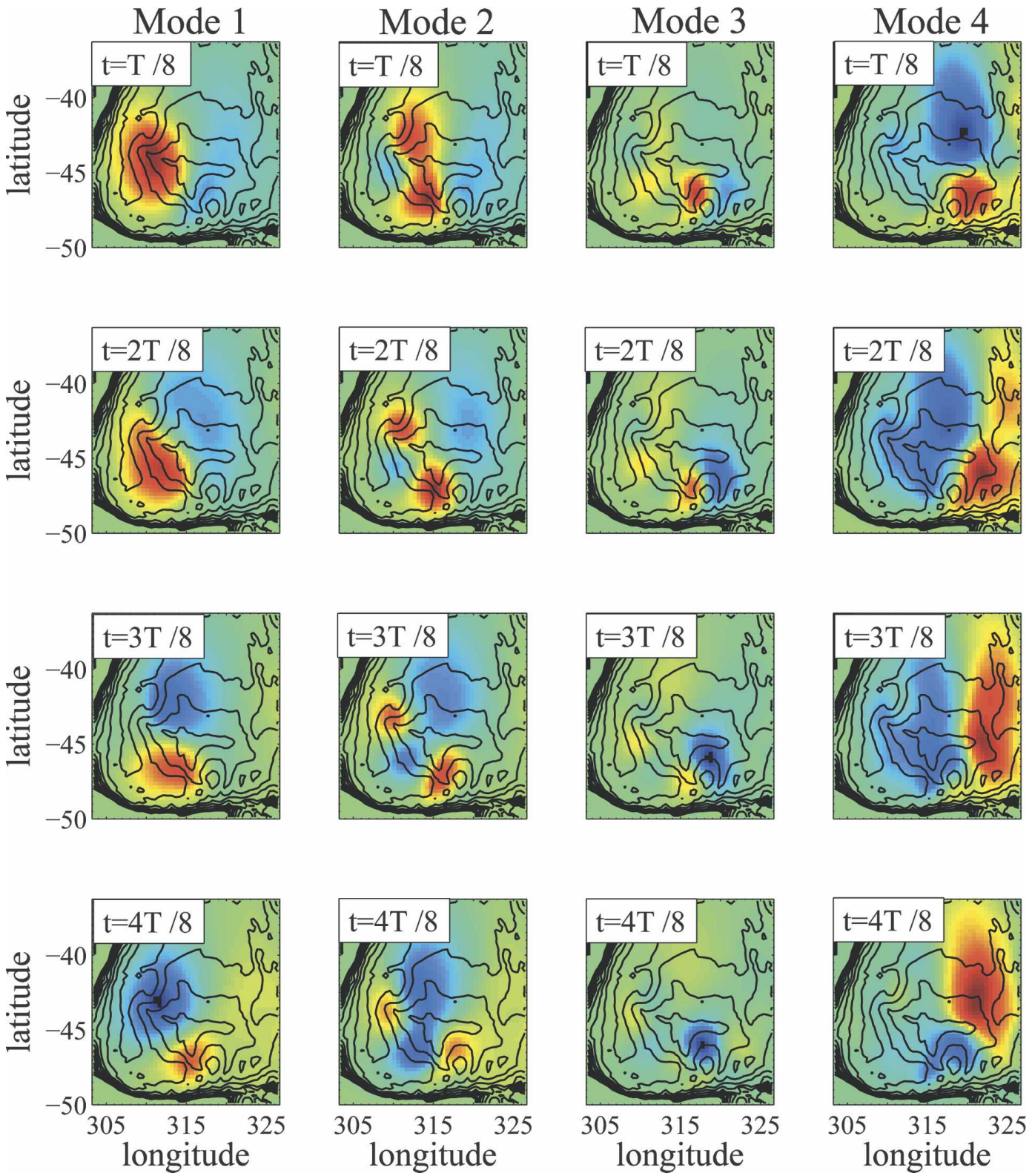

FIG. 3. Temporal evolution of modes 1-4 for the full-bathymetry case, zoomed in on the Zapiola Rise region. The four snapshots are 1/8th of a period apart and define one-half of an oscillatory cycle. Contours $\operatorname{show} \log (f / h)$, plotted for the interval $[-8.0,-7.5]$ with step 0.02 .

However, not all the modes could be traced throughout the $(\alpha, \beta)$ plane. As eigenvalue branches should be continuous in the parameters, these modes cannot disappear. The origin of this problem is a numerical one: the spatial scale of these modes becomes too small to resolve on the chosen grid and the JDQZ method cannot detect them anymore. This is the case for mode 3, which cannot be followed beyond $\beta=0.21$. Although its trace seems to end on the curve of mode 7 , a merging of these modes can be excluded, since their decay time scales do not converge.
In addition to the 6 numbered modes that were determined for $H_{\text {full }}$, a mode was found with a period of 41.6 days. This mode, called mode A, could not be followed beyond $\alpha>0.65$. It seems to originate from bathymetric features outside the Argentine Basin in the northeast corner of the model domain. In contrast to the other modes, its period (Fig. 4a) and spatial pattern are insensitive to the presence of bathymetry (measured by the parameter $\alpha$ ). The nature of this mode is not clear, but it does not appear to be a basin mode of the Argentine Basin. 
TABLE 2. Characteristics of dynamical modes $1-6 ; T_{\text {decay }}$ is the decay time scale (days) for standard parameter values; $T_{\text {full }}, T_{\text {flat }}$, and $T_{\text {rect }}$ denote the periods (days) of the modes for the fullbathymetry $\left(H_{\text {full }}, \alpha=0\right)$, flat-bottom $\left(H_{\text {flat }}, \alpha=1\right)$, and rectangular basin $\left(H_{\text {rect }}, \beta=1\right)$, respectively. The mode numbers of the corresponding rectangular Rossby basin mode are given by $(n, m)$, and $T_{n, m}$ is an estimate of its period based on (2); $T_{\mathrm{BG} 1}$ and $T_{\mathrm{BG} 2}$ are the oscillation periods for two different background flows.

\begin{tabular}{ccccccccc}
\hline \hline Mode & $T_{\text {decay }}$ & $T_{\text {full }}$ & $T_{\text {flat }}$ & $T_{\text {rect }}$ & $(n, m)$ & $T_{n, m}$ & $T_{\mathrm{BG} 1}$ & $T_{\mathrm{BG} 2}$ \\
\hline 1 & 3.0 & 19.6 & 21.6 & 20.3 & $(1,1)$ & 19.5 & 19.8 & 19.9 \\
2 & 2.9 & 22.7 & 34.0 & 35.4 & $(1,2)$ & 34.0 & 22.2 & 22.7 \\
3 & 2.7 & 27.7 & 61.1 & - & - & - & 26.5 & 27.0 \\
4 & 2.9 & 31.5 & 47.1 & 41.2 & $(2,2)$ & 38.6 & 32.9 & 32.8 \\
5 & 2.8 & 39.7 & 39.8 & 36.8 & $(3,1)$ & 35.5 & 41.8 & 40.6 \\
6 & 2.7 & 45.2 & 50.5 & 56.7 & $(2,3)$ & 52.8 & 49.0 & 46.7 \\
\hline
\end{tabular}

Starting from the other end of the transformation, the continuation of mode $\mathrm{C}$ [the $(4,1)$ basin mode] stalls at $\beta=0.66$. Mode B [the $(2,1)$ mode] could not be identified for $\beta=0$, and there is not a trace of this mode in the $\alpha$-segment. Inspection of the spatial patterns close to $\beta=0$ suggests that the mode is linked to activity on the relatively shallow areas outside the Argentine Basin. Modes 7-9 are high-order modes that could be traced back all the way to $\alpha=0$ (curves not shown), where they transform into long-period oscillations.

\section{Sensitivity}

\section{a. Sensitivity to domain size}

Basin boundaries play an essential role in the dynamics of classical basin modes. This raises the question of whether the limited domain size of the full-bathymetry case influences the spatial patterns and oscillation periods of the eigenmodes. To investigate this, we repeated the calculations for a larger domain, now stretching from $55^{\circ}$ and $20^{\circ} \mathrm{S}, 290^{\circ} \mathrm{E}$ to $360^{\circ}$.

The results (not shown) confirm that the modes are not influenced by the exterior domain boundaries: all modes are recovered with exactly the same spatial patterns, and with identical time scales. It is therefore clear that the relevant "boundaries" are provided by the natural geometry of the Argentine Basin (i.e., its almost closed contours of $f / H)$.

\section{b. Sensitivity to friction}

To investigate the sensitivity of the oscillation period and decay time scale to the specific choice of frictional parameters, we repeated the normal-mode analysis for different strengths of bottom friction $(r)$ and horizontal viscosity $\left(A_{h}\right)$; in particular the decay time scale and oscillation period of modes 1 and 4 are monitored (Fig. 5). In one set of computations $A_{h}$ is changed with fixed $r$ (Fig. 5b; black lines in Fig. 5a), in the other $r$ is decreased while keeping $A_{h}$ constant (Fig. 5c; gray lines in Fig. 5a). The most striking result is that bottom friction strongly controls the damping of the modes, but does not influence their oscillation periods. Lateral viscosity, on the other hand, mostly affects the oscillation period.

How much can we expect the oscillation periods to change when friction parameters are reduced from their standard values to the inviscid limit? Since $T_{\text {oscil }}$ is insensitive to $r$, we expect that reducing bottom friction will not lead to substantial changes in the period. In addition, Fig. 5b suggests that the periods are also reasonably well converged with respect to $A_{h}$. Since the modes are confined to the Argentine Basin and not influenced by $A_{h}$-dependent boundary layers at the
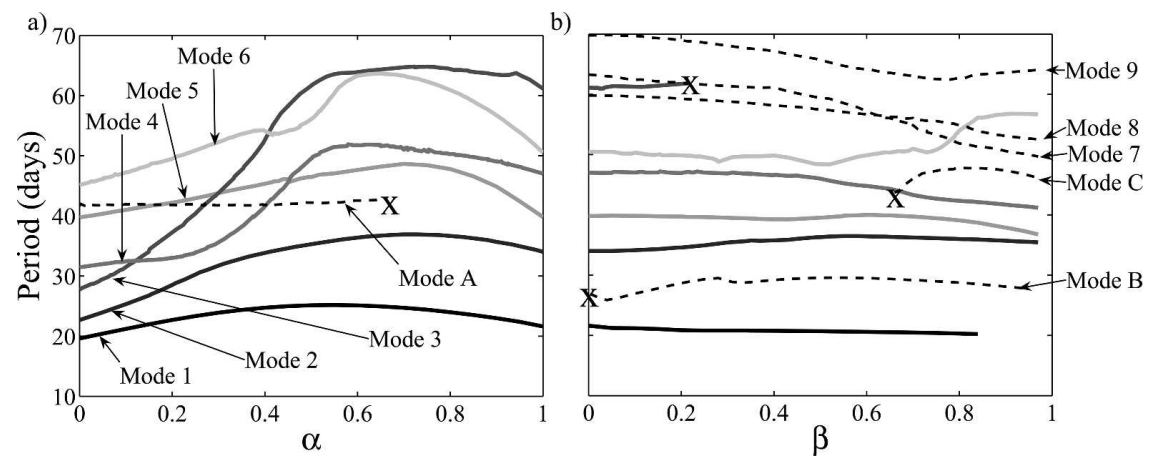

FIG. 4. The transition of eigenmodes from (a) the full-bathymetry $\left(H_{\text {full }}\right)$ to the flat-bottom Argentine Basin $\left(H_{\text {flat }}\right)$, as governed by $\alpha$, and (b) from $H_{\text {flat }}$ to the rectangular domain $\left(H_{\text {rect }}\right)$, as governed by $\beta$. The six fundamental modes with periods below 50 days (at $\alpha=0)$ are plotted. A cross indicates the position where a curve could not be followed any farther. Dashed line denotes other modes as discussed in the text. 
TABLE 3. Interpretation of modes $1-9$, and modes B and C, in terms of their mode number in zonal $(n)$ and meridional $(m)$ direction.

\begin{tabular}{ccccc}
\hline \hline$m \backslash n$ & 1 & 2 & 3 & 4 \\
\hline 1 & 1 & $\mathrm{~B}$ & 5 & $\mathrm{C}$ \\
2 & 2 & 4 & 8 & \\
3 & 7 & 6 & 9 & \\
\hline
\end{tabular}

sidewalls, one can be confident that no large changes in period occur below $A_{h}=500 \mathrm{~m}^{2} \mathrm{~s}^{-1}$.

The damping time scale of the modes, on the other hand, is strongly related to the value of the friction parameter $r$. The value chosen for the standard configuration (Table 1 ) is comparable to the best estimate of Hirose et al. (2001), who compared a global $1.125^{\circ}$ shallow-water model with altimeter observations. However, it is likely that the true value of $r$ (i.e., the value that is appropriate for this resolution and for this region) is much lower. Although the modes in this model configuration are strongly damped, it is shown in Part I that they are easily excited by stochastic forcing.

\section{c. Sensitivity to background flow}

To study the sensitivity of the modes with respect to a background circulation, we repeated the normalmode calculations for the six basin modes in the presence of background circulation. To represent the background circulation in the Argentine Basin, we use the barotropic velocity field of the U.K. high-resolution model OCCAM. Both the time-mean fields are used, as well as a particular snapshot that was chosen based on the presence of a particularly strong zonal current (Fig. 6).

The flow fields display a confluence of a northward flowing Malvinas Current and a southward flowing Brazil Current at $42^{\circ} \mathrm{S}$. A recirculation cell is visible in this region, as well as an eastward jet (South Atlantic Current) arising from the confluence. As in most models, an intense Zapiola anticyclone (Saunders and King 1995a; De Miranda et al. 1999) is absent.

The normal-mode calculations are repeated with either the time-mean (BG1) or the snapshot velocity field (BG2) as background flow. Technically, these velocity fields enter the equation as $\overline{\mathbf{x}}$ through the Jacobian matrix $\mathbf{N}_{x}$ in Eq. (8). The resulting oscillation periods are listed under $T_{\mathrm{BG} 1}$ and $T_{\mathrm{BG} 2}$ in Table 2. These results show that the modes and their oscillation periods are only slightly affected by the barotropic background circulation. The periods of modes 2 and 3 decrease slightly, while the periods of the other modes increase somewhat (up to $8 \%$ for mode 6 ). The characteristics of the spatial patterns of the modes remain intact.
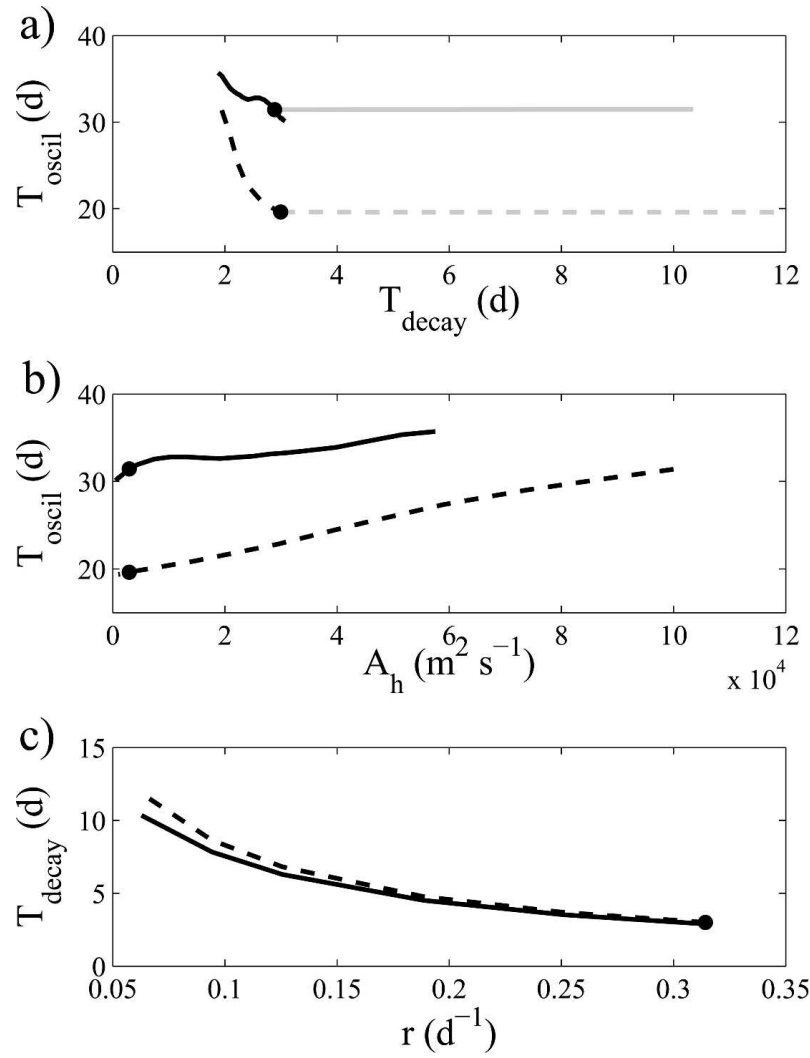

FIG. 5. Sensitivity of modes 1 (dashed lines) and 4 (solid lines) with respect to friction parameters $r$ and $A_{h}$. (a) Oscillation period and decay time scale for computations where only $A_{h}$ is changed (black lines) or $r$ (gray lines). (b) Oscillation time scale as function of $A_{h}$. Curves correspond to black lines in (a). (c) Decay time scale as function of $r$. Curves correspond to gray lines in (a). Black dots denote the solution corresponding to the standard values of Table 1 .

Since the OCCAM model does not represent the Zapiola anticyclone, an additional analysis was performed to study the sensitivity of modes 1 and 4 with respect to an anticyclonic circulation around the Zapiola Rise. To represent this anticyclone, a Gaussian high-pressure region was constructed, centered at $45^{\circ} \mathrm{S}, 317.5^{\circ} \mathrm{E}$, with a $5^{\circ}$ half-width. A flow field was prescribed to be in geostrophic balance with this sea surface elevation. Characteristic flow velocities in this region are of the order of $0.1 \mathrm{~m} \mathrm{~s}^{-1}$ (Saunders and King 1995b). Given the characteristics of the Gaussian shape, this is achieved by a sea surface elevation of about $0.5 \mathrm{~m}$. Assuming a water depth of $5000 \mathrm{~m}$, this corresponds to a transport of $230 \mathrm{~Sv}\left(1 \mathrm{~Sv} \equiv 10^{6} \mathrm{~m}^{3} \mathrm{~s}^{-1}\right)$. It is clear that this estimate is on the high side of possible values.

The anticyclone indeed reduces the oscillation periods of the two dominant modes 1 and 4 , as can be expected from advection of anticyclonic modes by an anticyclonic circulation. The period of mode 1 is re- 


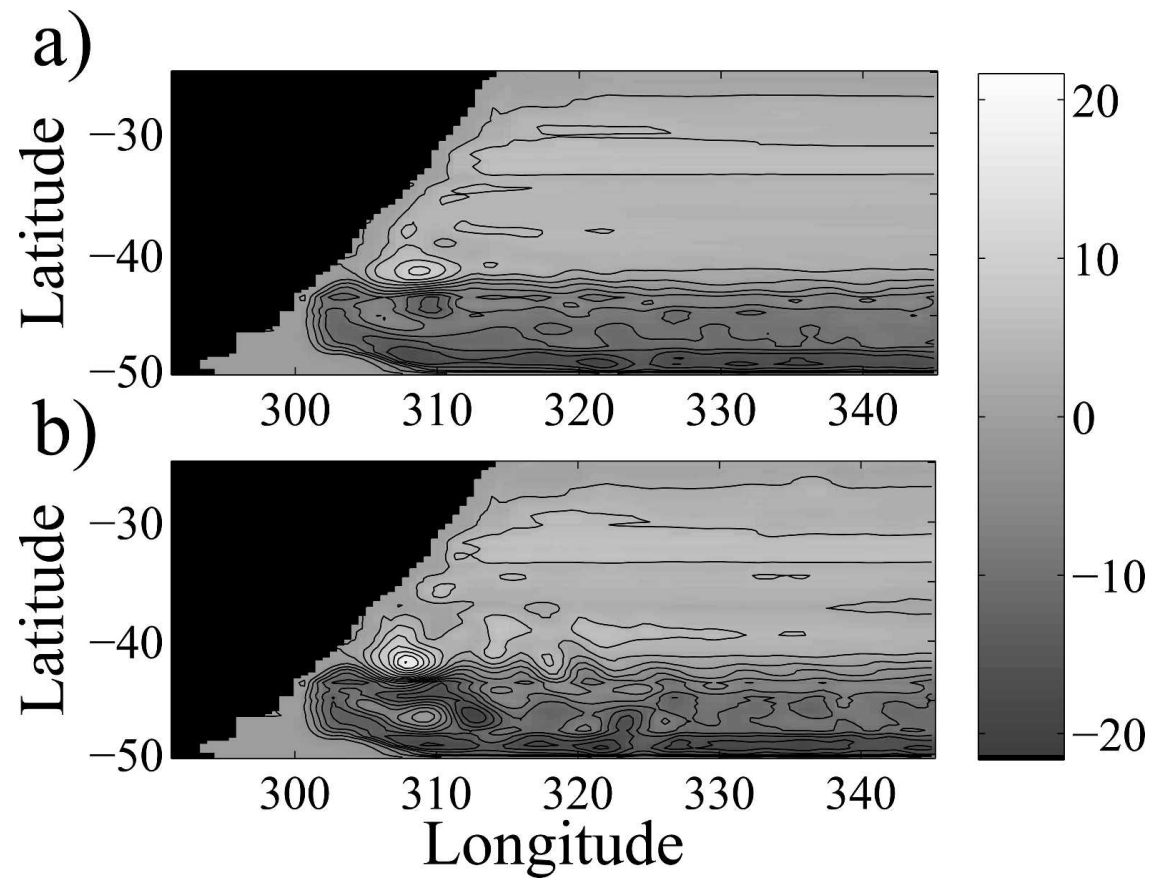

FIG. 6. Impression of (a) the time-mean and (b) a snapshot of the streamfunction of the barotropic flow in the Argentine Basin from the high-resolution model OCCAM. Streamlines are plotted at a 2.5 -Sv interval. OCCAM barotropic velocity fields are interpolated onto our model grid. Streamfunction is calculated by integrating the interpolated meridional velocity vertically and eastward, using the bathymetry of the SW model. Because of small discrepancies in bathymetry between OCCAM and this model, and interpolation errors, the barotropic transport is not fully divergence free. No efforts have been made to correct for this, since this would have made a negligible impact on the normal-mode calculations.

duced from 19.6 to 18.5 days, whereas the 31.5 days of mode 4 is reduced to 29.7 .

\section{Discussion and conclusions}

An unbounded, flat-bottom (constant depth) ocean on a rotating sphere supports a continuous spectrum of Rossby waves, which are controlled by the gradient in background potential vorticity $f / H$. When meridional boundaries are taken into account, a discrete spectrum of modes arises, so-called Rossby basin modes (Longuet-Higgins 1964). Many studies have shown that bathymetry drastically affects the propagation characteristics of Rossby waves and basin modes (e.g., Rhines 1969; Anderson and Killworth 1977; Ripa 1978; Platzman 1975; Platzman et al. 1981; Miller 1986; Miller et al. 1987; Miller 1989). In fact, Koblinsky (1990) showed that there are relatively few places in the World Ocean where the planetary vorticity gradient $\beta_{0}$ is the dominant component of the potential vorticity gradient.

Nonetheless, classical theory may still be important for determining the spectrum of modes in realistic basins. As outlined in the introduction, bathymetry ex- pands the vorticity balance of a homogeneous fluid with a topographic- $\beta$ effect, $\beta_{T}=-f \boldsymbol{\nabla} \ln H$. For reasonable choices of bathymetry, a smooth transition between the full-bathymetry and flat-bottom cases can be defined that leaves the spectrum of eigenmodes intact. In this paper a connection was established between the spectrum of barotropic basin modes of the Argentine Basin and the classical basin modes in a flat-bottom, rectangular domain. The Argentine Basin modes can therefore be interpreted as vortical modes that are controlled by the gradient in background potential vorticity $f / H$.

Miller et al. (1996) were the first to unambiguously observe a topographic Rossby mode, which propagated along the Iceland-Faeroe Ridge between the Faeroe Islands and Iceland. More recently, Beckenbach and Washburn (2004) observed a Rossby mode in the Santa Barbara Channel, which appeared to be governed by a rather uniform tilt in seafloor. The interpretation of these modes in terms of vorticity was facilitated by a relatively uniform distribution of $f / H$ contours, and a relatively small domain size.

The bathymetry of the Argentine Basin is severely 


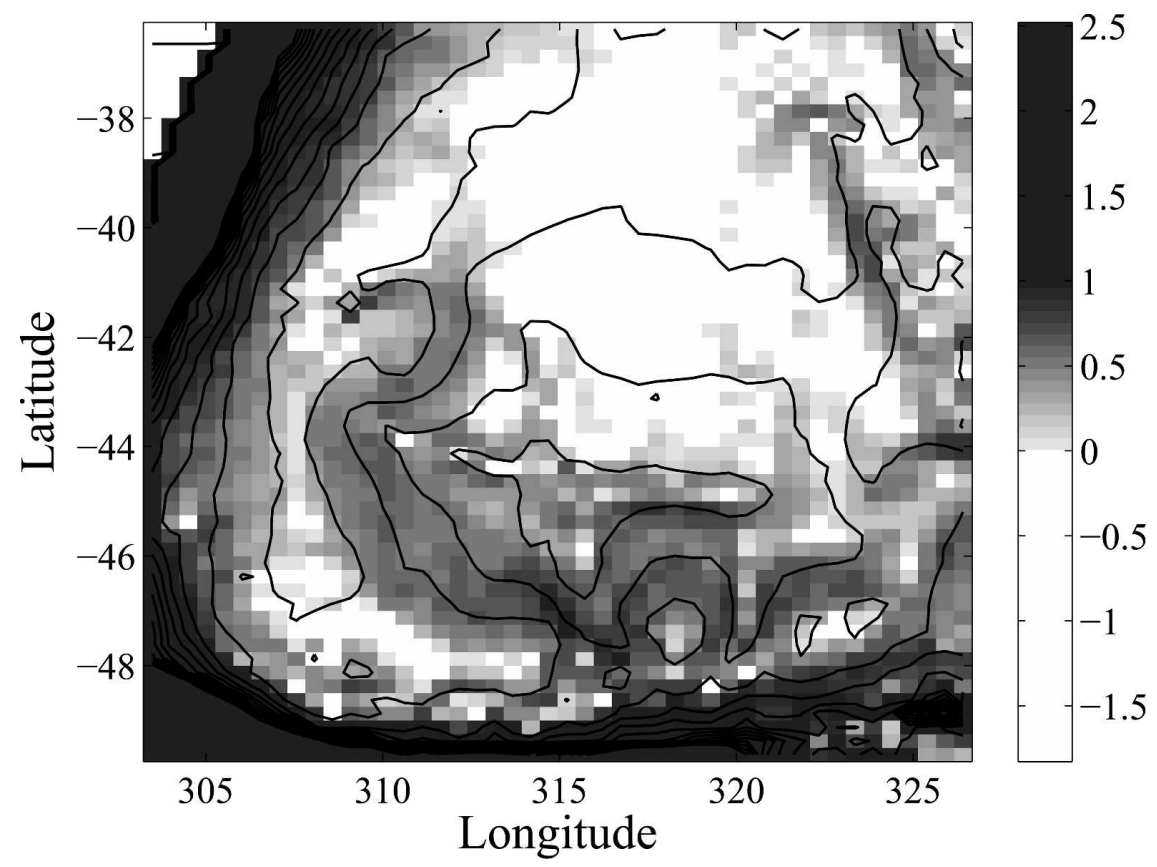

FIG. 7. Base-10 logarithm of the ratio between the gradient of "topographic" vorticity $|f \nabla \ln H|$ and its planetary counterpart $\beta_{0}$. Positive values mean a ratio $>1$ and hence a dominance of the topographic over the planetary component. Values exceeding 1 are found only on the continental slope. Contours show $\log (f / h)$, plotted for the interval $[-8.0,-7.5]$ with step 0.02 .

complicated by the presence of the Zapiola Rise, which leads to strongly convoluted contours of $f / H$. The impact of this seamount is clearly illustrated by the white contours in Fig. 1. Nonetheless, the Argentine Basin is large enough that the gradient of planetary vorticity is not negligible (Fig. 7). Although the gradient of $f / H$ on the southern flank of the Zapiola Rise is clearly dominated by the topographic component, there are extensive areas in the Argentine Basin, most notably the northern half and the southwest corner, where the planetary component is dominant. In this context it is not straightforward which of the full-bathymetry modes portrayed in Fig. 2 (left columns) should be considered to be the fundamental mode, nor how many low-order modes there should exist. Here the explicit connection to the spectrum of classical modes is useful to interpret the full-bathymetry modes.

It could be argued that the full-bathymetry modes are governed by local topography only, and have no significance for the entire basin. Obviously it is hard to establish in detail how the mode selection takes place, and why, for instance, the large-scale rotating mode 1 is related to the $(1,1)$ classical mode, and mode 4 to the $(2,2)$ mode, while both of them seem to be a fundamental mode on the local bathymetry (the semicircular contours of $f / H$ on the west and east side of the Zapiola Rise, respectively; Fig. 3). However, Fig. 4 shows (i) that the connections exist, and (ii) that there is a general tendency for the lowest-order modes of the full-bathymetry basin to be related to the lowest-order modes of classical theory, while higher-order modes tend to retain their high-wavenumber character.

As a matter of fact, studies like Miller et al. (1996), Beckenbach and Washburn (2004), and Pierini (1996) apply a similar transformation of a flat-bottom basin to a rectangular domain to identify the fundamental mode in their observations or modeling results. They hence implicitly assume an equivalence between this mode in their real ocean domain and in a rectangular basin with homogeneously distributed contours of $f / H$. In this study this equivalency is taken a step further by not only assuming equivalence of a rectangular basin $\left(H_{\text {rect }}\right)$ with a flat-bottom basin with realistic topography $\left(H_{\text {flat }}\right)$, but also with a basin with full bathymetry. The idea that the spectrum of classical Rossby modes remains invariant under transformation to a real basin-albeit with sometimes strong modifications to the spatial patterns and oscillation periods of the individual modes - may guide future studies addressing the barotropic spectrum of (semi)enclosed basins, especially those for which the planetary vorticity gradient is not negligible.

Note that the transition from basin-scale modes to modes captured by localized topography is often ac- 
companied by counterbalancing effects on the oscillation period (Ripa 1978). The trend toward smaller scales tends to increase the oscillation period, while the trend toward often higher gradients of ambient potential vorticity (due to the dominance of topographic effects) tends to reduce it. For almost all modes presented in Table 2 the latter effect appears to dominate.

Except for mode 3, all of the modes 1-6 could be traced back to classical modes. However, not all of the classical modes could be followed in the reverse direction to the realistic basin. In particular, the fact that the low-order $(2,1)$ mode B could not be followed all the way to $H_{\text {full }}$ might appear somewhat worrisome, since its oscillation time scale is of the right order when compared to the 25-day oscillation found in observations (Fu et al. 2001). However, its large-scale structure disappeared when approaching $H_{\text {flat }}$ and its focus shifted to the shallow areas outside the basin. No trace was found of this mode in the $\alpha$ segment of the domain transformation (connecting $H_{\text {full }}$ and $H_{\text {flat }}$ ), despite extensive calculations. For reasons that are not fully understood, this mode does not seem to correspond to a basin mode of the full-bathymetry Argentine Basin.

A sensitivity study with respect to friction shows that the decay rate of the modes is strongly controlled by bottom friction, but not at all by horizontal viscosity. Given the large uncertainties in the amplitude of bottom friction, it is clear that the decay rates determined here are not well constrained. The oscillation period of the modes, on the other hand, appears to be affected mainly by horizontal viscosity. Based on Fig. 5b, it seems reasonable to conclude that the base value of $A_{h}$ used in this study $\left(3.0 \times 10^{3} \mathrm{~m}^{2} \mathrm{~s}^{-1}\right)$ is small enough to assume that the oscillation periods of the eigenmodes are determined with reasonable accuracy.

The analysis in section 3 showed that the modes were scarcely affected by the barotropic circulation of OCCAM, or by a strong Zapiola anticyclone. This may not be surprising when comparing the large spatial scale of the modes $\left[O\left(10^{5}-10^{6} \mathrm{~m}\right)\right]$ and their relatively short time scale (20-30 days), with the typical width $\left[O\left(10^{4}\right.\right.$ $\mathrm{m})]$ and velocity $\left[O\left(10^{-1} \mathrm{~m} \mathrm{~s}^{-1}\right)\right]$ of oceanic jets like the South Atlantic Current (Stramma and Peterson 1990) or the Zapiola anticyclone (Saunders and King 1995b).

The most important control on the modes is exerted by bathymetry. This may be considered good news for modelers, since in most areas of the World Ocean bathymetry is better known than the mechanisms of frictional decay, especially on the larger spatial scales that are important for barotropic modes. This finding also shows, however, that care should be taken when smoothing bathymetry. Bathymetric smoothing is most often applied in low-resolution models to assure numerical stability. However, it flattens out bathymetry in much the same way that increasing $\alpha$ flattens out the Argentine Basin in this study. As an example, Hughes et al. (2007) discuss the variability in the sigmacoordinate model used by De Miranda et al. (1999), and found an oscillation with a period of about 22 days. The spatial pattern of the dominant CEOF clearly displays the plane-wave propagation characteristic of classical basin modes. This suggests that filtering bathymetry has reduced the topographic features and put the model in the domain $\alpha>0$. Yet, it also confirms the conclusion of Part I that these models really reproduce the fundamental barotropic basin mode, but not the 25-day mode.

In this study, the basin modes were traced through parameter space in a rather ad hoc fashion, as many modes were calculated for closely spaced parameter values. In most cases the connection between modes could unambiguously be determined, since both the growth rate and oscillation period of the modes change smoothly with $\alpha$ and $\beta$. However, in a few cases modes were "lost" beyond certain parameter values. This was attributed to the modes developing spatial scales that are too small to resolve. Possibilities to apply parameter continuation techniques to follow eigenmodes through parameter space are currently being considered for implementation.

Acknowledgments. This research was supported by NSF through Grant 0424703 (WW, SG, and HD), by NASA JPL Contract 1224031 (SG), and by the Climate Change Prediction Program of the U.S. Department of Energy Office of Science (WW); FV is supported by CNES and CNRS. Los Alamos National Laboratory is operated by the Los Alamos National Security LLC for the National Nuclear Security Administration of the U.S. Department of Energy under Contract DE-AC5206NA25396. Barotropic velocity data of the OCCAM model were kindly made available by Andrew Coward and Beverly de Cuevas (National Oceanography Centre, Southampton). This paper benefited from the comments of two anonymous reviewers.

\section{REFERENCES}

Anderson, D. L. T., and P. D. Killworth, 1977: Spin-up of a stratified ocean, with topography. Deep-Sea Res., 24, 709-733.

Beckenbach, E., and L. Washburn, 2004: Low-frequency waves in the Santa Barbara Channel observed by high-frequency radar. J. Geophys. Res., 109, C02010, doi:10.1029/ 2003JC001999.

De Miranda, A. P., B. Barnier, and W. K. Dewar, 1999: On the dynamics of the Zapiola anticyclone. J. Geophys. Res., 104, 21 137-21 149. 
Dijkstra, H. A., 2006: On the interaction of SST modes in the North Atlantic Ocean. J. Phys. Oceanogr., 36, 286-299.

Fu, L.-L., B. Cheng, and B. Qiu, 2001: 25-day period large-scale oscillations in the Argentine Basin revealed by the TOPEX/ Poseidon altimeter. J. Phys. Oceanogr., 31, 506-517.

Hirose, N., I. Fukumori, and V. Zlotnicki, 2001: Modeling the high-frequency barotropic response of the ocean to atmospheric disturbances: Sensitivity to forcing, topography, and friction. J. Geophys. Res., 106, 30 987-30995.

Hughes, C. W., V. N. Stepanov, L.-L. Fu, B. Barnier, and G. W. Hargreaves, 2007: Three forms of variability in Argentine Basin ocean bottom pressure. J. Geophys. Res., 112, 1464, doi:10.1029/2006JC003679.

Jin, F.-F., and J. D. Neelin, 1993: Modes of interannual tropical ocean-atmosphere interaction-A unified view. I: Numerical results. J. Atmos. Sci., 50, 3477-3503.

Koblinsky, C. J., 1990: The global distribution of $f / H$ and the barotropic response of the ocean. J. Geophys. Res., 95, 3213-3218.

Longuet-Higgins, M. S., 1964: Planetary waves on a rotating sphere. Proc. Roy. Soc. London A, 279, 446-473.

Miller, A. J., 1986: Nondivergent planetary oscillations in midlatitude ocean basins with continental shelves. J. Phys. Oceanogr., 16, 1914-1928.

- 1989: On the barotropic planetary oscillations of the Pacific. J. Mar. Res., 47, 569-594.

— - W. R. Holland, and M. C. Hendershott, 1987: Open-ocean response and normal mode exitation in an eddy-resolving general circulation model. Geophys. Astrophys. Fluid Dyn., 37, 253-278.

— , P. F. J. Lermusiaux, and P.-M. Poulain, 1996: A topographic-Rossby mode resonance over the Iceland-Faeroe ridge. J. Phys. Oceanogr., 26, 2735-2747.

Pedlosky, J., 1987: Geophysical Fluid Dynamics. Springer-Verlag, $710 \mathrm{pp}$.
Pierini, S., 1996: Topographic Rossby modes in the Strait of Sicily. J. Geophys. Res., 101, 6429-6440.

Platzman, G. W., 1975: Normal modes of the Atlantic and Indian Oceans. J. Phys. Oceanogr., 5, 201-221.

— , G. A. Curtis, K. S. Hansen, and R. D. Slater, 1981: Normal modes of the World Ocean. Part II: Description of modes in the period range 8 to 80 hours. J. Phys. Oceanogr., 11, 579603.

Rhines, P. B., 1969: Slow oscillations in an ocean of varying depth. Part I: Abrupt topography. J. Fluid Mech., 37, 161-189.

Ripa, P., 1978: Normal Rossby modes of a closed basin with topography. J. Geophys. Res., 83, 1947-1957.

Saunders, P. M., and B. A. King, 1995a: Oceanic fluxes on the WOCE A11 section. J. Phys. Oceanogr., 25, 1942-1958.

_ , and _ 1995b: Bottom currents derived from shipborne ADCP on WOCE cruise A11 in the South Atlantic. J. Phys. Oceanogr., 25, 329-347.

Schmeits, M. J., and H. A. Dijkstra, 2000: Physics of the 9-month variability in the Gulf Stream region: Combining data and dynamical systems analysis. J. Phys. Oceanogr., 30, 19671987.

Simonnet, E., and H. A. Dijkstra, 2002: Spontaneous generation of low-frequency modes of variability in the wind-driven ocean circulation. J. Phys. Oceanogr., 32, 1747-1762.

Sleijpen, G. L. G., and H. A. Van der Vorst, 1996: A JacobiDavidson iteration method for linear eigenvalue problems. SIAM J. Matrix Anal. Appl., 17, 410-425.

Stramma, L., and R. G. Peterson, 1990: The South Atlantic Current. J. Phys. Oceanogr., 20, 846-859.

Weatherly, G. L., 1993: On deep-current and hydrographic observations from a mudwave region and elsewhere in the Argentine Basin. Deep-Sea Res. II, 40, 939-961.

Weijer, W., F. Vivier, S. T. Gille, and H. A. Dijkstra, 2007: Multiple oscillatory modes of the Argentine Basin. Part I: Statistical analysis. J. Phys. Oceanogr., 37, 2855-2868. 